\title{
General sessions
}

DNA replication and chromosome segregation (1A-01 1A-09)

1 A KEYAMURA, Kenji ${ }^{1}$, SU'ETSUGU, Masayuki ${ }^{1}$, UEDA, -01 Tadashi ${ }^{2}$, KATAYAMA, Tsutomu ${ }^{1}$ ( ${ }^{1}$ Dept. Molec. Biol., Kyushu Univ. Grad. Sch. Pharm. Sci., ${ }^{2}$ Dept. Immun., Kyushu Univ. Grad. Sch. Pharmceutical Sci.)

Molecular mechanism of the sliding clamp-dependent inactivation of DnaA in $E$. coli: Roles for the DnaA DNA-binding domain

In Escherichia coli, the ATP-bound DnaA protein initiates chromosomal replication from oriC. After initiation, DnaA-bound ATP is hydrolyzed in a manner depending on Hda protein and the DNA-loaded sliding clamp of the DNA polymerase III holoenzyme, which yields ADP-DnaA, the inactivated form for initiation. This mechanism is termed RIDA (Regulatory inactivation of DnaA) system, which represses the DnaA activity to prevent overinitiation. So far, to analyze molecular mechanisms and structure-function relationship in RIDA, we have used mutant DnaA proteins that bear an amino-acid substitution in the AAA+ (ATP binding/hydrolysis) domain. In this study, we report that purification and functional analysis of three kinds of DNAbinding domain mutants of DnaA. Roles for this domain in RIDA system will be discussed.

1 A OZAKI, Shogo ${ }^{1}$, FUJIMITSU, Kazuyuki ${ }^{1}$, KEYAMURA, -02 Kenji ${ }^{1}$, KAWAKAMI, Hironor ${ }^{1}$, SU'ETSUGU, Masayuki ${ }^{1}$, KURUMIZAKA, Hitoshi ${ }^{2}$, KATAYAMA, Tsutomu ${ }^{1}\left({ }^{1}\right.$ Dept. Molec. Biol., Kyushu Univ. Grad. Sch. Pharm. Sci., ${ }^{2}$ Sch. Sci. Eng., Waseda Univ.)

Analysis of the initiation activity by hyperthermophilic eubacterium Thermotoga maritima DnaA homolog

Escherichia coli DnaA protein, the initiator of chromosomal replication, binds to a specific 9-mer sequence (DnaA box) in the chromosomal replication origin (oriC). Minimum oriC contains five DnaA boxes and three AT-rich 13-mer repeats. A complex consisting of minimum oriC and multimeric ATP-bound DnaA molecules leads to local unwinding of the 13-mer region. This event is crucial in the initiation steps of DNA replication. However, high order structure of the nucleoprotein complex remains unknown. As $E$. coli DnaA protein is easily denatured by heat and forms irregular aggregates under low salt conditions, we started to analyze hyperthermophilic eubacterium Thermotoga maritima MSB8 DnaA homolog (TmaDnaA). Using purified TmaDnaA, we found that this protein is heat-resistant and has high affinity for ATP and ADP. Gel-shift assay indicated that TmaDnaA specifically binds to a 12 -mer specific DNA sequence that is seen repeatedly in the putative oriC region of this bacteria. These characters regarding binding to nucleotides and a specific sequence are similar to those observed for $E$. coli DnaA. Structural analysis of this protein is in progress.
1A HAN, Man ${ }^{1}$, YAGURA, Masaru ${ }^{1}$, AOKI, Kazuteru ${ }^{1}$, -03 ITOH, Tateo ${ }^{1}\left({ }^{1}\right.$ Dept. Biol., Faclt. Sci., Univ. Shinshu)

Molecular mechanism of early steps of ColE2 plasmid replication initiation

The initiator (Rep) protein of plasmid ColE2 specifically binds to the replicator (Ori), synthesizes a primer, unwinds doublestranded DNA and interacts with PolI during the course of initiation of plasmid DNA replication. In order to elucidate the early steps of replication initiation by Rep, we investigated the interaction between Rep and Ori, and the Ori-DNA melting by Rep. We identified the binding domains in Rep and the corresponding binding sites in Ori important for Rep-Ori interaction. The interaction between Rep and the region of Ori containing the primer sequence is necessary for unwinding of DNA strands in Ori, although this interaction did not induce significant bending of Ori DNA. It is suggested that this interaction induces local destabilization of double-stranded DNA and that the single-stranded DNA binding domain of Rep binds to the single-stranded region containing the primer sequence to stabilize the unwound structure. All these sequential events might enable the primase domain of Rep to synthesize the primer RNA at the specific site on the template strand.

1 A FURUYA, Nobuhisa ${ }^{1}$, KOMANO, Teruya ${ }^{1}\left({ }^{1}\right.$ Dept. -04 Biol., Tokyo Metro. Univ.)

Determination of the nick site at oriT of conjugative plasmid R721.

Conjugative transfer of bacterial plasmid such as F, R64 and R721 is initiated by site- and strand-specific cleavage (nicking) into the origin of transfer, oriT. At the oriT site, protein-DNA complex called relaxosome is formed within bacterial cells. R721 is a conjugative plasmid belonging to the incompatibility group I2. Elucidation of the genome sequence of $R 721$ had revealed that, while its oriT structure is similar to that of IncI1 and IncP plasmids, its relaxosome component proteins are related to that of non-conjugative but mobilizabe plasmids such as ColE1. The relaxosome proteins essential for R721 conjugative transfer, NikA and NikB, are not related to that of R64 NikA and NikB, respectively. The nick region sequences among IncP, IncI1, and other related plasmids share a highly conserved 6 -bp sequence (ATCCTG) at the 3' side of the cleavage site. However, a sequence, ATCGTG, was found within the corresponding site within the R721 oriT. To examine the cleavage site within R721 oriT, the nicked R721 oriT plasmid was isolated and the cleavage site was determined. The cleavage has been introduced into a site one-nucleotide more downstream than the postulated one (ATCGTGC/). 
1A ADACHI, Shun ${ }^{1}$, HIRAGA, $\operatorname{Sota}^{2}\left({ }^{1} \mathrm{Lab}\right.$. Immun. Cell -05 Biol., Grad. Sch. BioStud., Kyoto Univ., ${ }^{2}$ Lab. Chrom. Transmission, Grad. Sch. BioStud., Kyoto Univ.)

Spiral-shaped localization of F plasmid SopA and SopB proteins: Implications in new modeling of plasmid positioning and partitioning

F plasmid DNA initially locates at mid-cell during cell cycle in $E$. coli cells growing in poor media. After replication sister copies migrate to $1 / 4$ and $3 / 4$ cellular positions and then the cell divides. The proper positioning of $\mathrm{F}$ plasmid requires the segregation machinery controlled by the sop $A B C$ system. The sop $A B C$ system is analogous to the chromosome segregation machinery parABS system, which many plasmids and bacteria such as P1 plasmid or B. subtilis possess. We have found that SopA localizes as spiralshaped structures in the cell like ParA of another plasmid. Although SopB locates as fluorescent foci on plasmid DNA, we have found that SopB localizes as spiral-shaped structures in plasmid-free segregant cells in SopA-dependent manner. The latter fact is inconsistent with the assumption of the model proposed by Hunding et al. (2003), therefore we have to consider another model.
1A TOHRI, Kazuo ${ }^{1}$, FUJIKANE, Ryosuke ${ }^{1,2}$, YAMAGAMI, -07 Takeshi ${ }^{1}$, ISHINO, Yoshizumi ${ }^{1}$ ( ${ }^{1}$ Dev. Genetic Resources Tech., Univ. Kyushu, ${ }^{2}$ Dept. Mol. Microbiol. Res. Inst. Microb. Dis., Univ. Osaka)

Functional and structural correlations of family D DNA polymerase from hyperthermophilic archaeon, Pyrococcus furiosus

DNA polymerases are classified into six family (family A, B, C, D, $\mathrm{X}$, and $\mathrm{Y}$ ). In archaea, crenarchaeal organisms have at least two family B enzymes and a family Y enzyme. On the other hand, euryarchaeal organisms have one family B DNA polymerase and a family D DNA polymerase, which is very unique to euryarchaeota among living organisms.

Pol D is composed of two subunits, DP1 (69 kDa) and DP2 (143 $\mathrm{kDa}$ ), and the latter is catalytic subunit. Based on the biochemical analysis, $P$. furiosus Pol $D$ has a very excellent primer extension activity and a strong 3'-5' exonuclease activity in vitro, and complex formation of DP1 and DP2 is essential for these activities. DP1 has some sequence similarity to that of the second subunits of the eukaryotic replicases(Pol delta and epsilon). However precise functions of this subunit remains unclear. To analyze functional and structural relationship of DP1 in the Pol D function, we made deletion mutant DP1 proteins and tried to identify essential regions for the functional interaction with DP2.

Biochemical properties of the mutant Pol D containing truncated DP1 subunits will be presented and structure-function relationships of DP1 will be discussed.

1A FURUKAWA, Nobuhiro ${ }^{1}$, FUJIKANE, Ryosuke ${ }^{1,2}$ -08 SHINAGAWA, Hideo ${ }^{2}$, ISHINO, Yoshizumi ${ }^{1}\left({ }^{1}\right.$ Lab. Genet. Prot. Eng., Univ. kyushu, ${ }^{2}$ Univ. Osaka)

Hef converts substrate preference by interaction with pcnas

Archaeal Hef endonuclease has structural homology to XPF/ Mus81 family nucleases and shows substrate preference for forkstructured DNA. Therefore, the main function of Hef is predicted to collapse the stalled fork, which is followed by homologous recombination for rebuilding fork structure. Hef of Aeropyrum pernix, which belongs to crenarchaeota, has a motif sequence for PCNA binding and some functional interactions between these proteins are predicted. As A. pernix has three PCNA homologues, we examined the interactions between each PCNA and Hef. Our analyses including enzyme assay and physical interaction indicate that Hef converts substrate specificity by changing PCNA partner among three PCNAs and function in the different types of repair systems. 
1 A UENO, Masaru ${ }^{1}$, KANG, Ho-young ${ }^{2}$, SEO, Yeon-soo ${ }^{2}$, -09 TOMITA, Kazunori ${ }^{1,3}$ ( ${ }^{1}$ Dept. Chem., Shizuoka Univ., ${ }^{2}$ Korea Adv. Inst. Sci. Tech, ${ }^{3}$ Cancer Res. UK)

Fission yeast dna2 is required for generation of the telomeric single-strand overhang

The S. pombe Rad50 (Rad50-Rad32-Nbs1) complex has been suggested to be required for the resection of the C-rich strand at telomere ends in taz1-d cells. However, the nuclease-deficient Rad32-D25A mutant can still resect the C-rich strand, suggesting the existence of a nuclease that resects the C-rich strand. Here, we demonstrate that a taz1-d dna2-2C double mutant lost the Grich overhang at a semi-permissive temperature. The amount of G-rich overhang in S phase in the dna2-C2 mutant was lower than that in wild-type cells at the semi-permissive temperature. Dna2 bound to telomere DNA in a ChIP assay. Moreover, telomere length decreased with each generation after shift of the dna2-2C mutant to the semi-permissive temperature. These results suggest that Dna2 is involved in the generation of G-rich overhangs in both wild-type cells and taz1-d cells. The dna2-C2 mutant was not g-ray sensitive at the semi-permissive temperature, suggesting that the ability to process DSB ends was not affected in the dna2-C2 mutant. Our results reveal that DSB ends and telomere ends are processed by different mechanisms.

\section{DNA recombination (1A-10 - 1A-24)}

1 A YAMAMOTO, $\mathrm{Kazuo}^{1}$, HIROUCHI, Tokuhisa ${ }^{1}$, -10 YAMAMOTO, Ayumu ${ }^{1}$, TERANISHI, Mika ${ }^{1}$, HIDEMA, Jun ${ }^{1}$, KUMAGAI, Tadashi ${ }^{1}{ }^{1}$ Grad. Sch. Life Sci.s, Tohoku Univ.)

\section{Class II DNA photolyase gene from Oriza sativa}

UV radiation induces two classes of photoproducts in DNA; the cyclobutane pyrimidine dimer (CPD) and the pyrimidine [6-4] pyrimidone photoproduct (6-4 product). Many organisms produce enzymes, termed photolyases, that specifically bind to these lesions and split them via a UV-A/blue light-dependent mechanism, reversing the damage. These photolyases are specific for either CPDs or 6-4 products. Two classes of photolyases (class I and class II) repair CPDs. A gene that expresses a protein with class II CPD photolyase activity in vitro has been cloned from plants including Arabidopsis thaliana and Cucumis sativus. We present the isolation of a homolog of this gene from the crop Oryza sativa. The cDNA constitutes a GC-rich (75\%) 5' region and a 50\% GC-containing 3 ' region. This gene produces a protein with CPD photolyase activity when expressed in $E$. coli. The CPD photolyase gene encodes at least two types of mRNA formed by alternative splicing of exon 5. One of the mRNAs encodes an ORF for 506 amino acid residues and the other for 364 amino acid residues.
1 A TERADA, Rie ${ }^{1}$, SAITOH, Miho ${ }^{1}$, JOHZUKA-11 HISATOMI, Yasuyo ${ }^{1}$, IIDA, Shigeru ${ }^{1}$ ( ${ }^{1}$ Div. Molec. Genet., Natl. Inst. Basic Biol.)

Targeting of the Adh1 and Adh2 genes by homologous recombination in rice

Rice is an important staple food and is also a model plant for other cereal species. Using an efficient and straightforward positive-negative selection procedure, we have repeatedly succeeded in obtaining mutants that had their endogenous Waxy genes modified precisely by homologous recombination. Since the strategy we adapted is independent of gene-specific selection, it must be applicable to genes other than the Waxy gene. To test the general applicability of our gene targeting strategy, we have tried to obtain mutants having the Adh1 and Adh2 genes for alcohol dehydrogenases modified separately by homologous recombination. Both Adh1 and Adh2 genes reside on chromosome 11 in rice and highly repetitive retroelements are present adjacent to these gene. We will report the characterization of mutants, in which either Adh1 and Adh2 gene has modified.

1A HAN, Yong-woon ${ }^{1}$, HISHIDA, Takashi ${ }^{2}$, TANI, -12 Tomomi $^{1}$, IWASAKI, Hiroshi ${ }^{3}$, HARADA, Yoshie ${ }^{1}$, SHINAGAWA, Hideo ${ }^{2}$ ( ${ }^{1}$ Dept. Molec. Physiology, Tokyo Metro. Inst. Med. Sci., ${ }^{2}$ Dept. Molec. MicroBiol., Res. Inst. Microb. Dis., Osaka Univ., ${ }^{3}$ Div. Molec. Cell. Biol., Grad. Sch. Integr. Sci., Yokohama City Univ.)

Direct observation of the RuvA-RuvB mediated branch migration of Holliday junction DNA with single-molecule analysis

Escherichia coli RuvA-RuvB protein complex promotes branch migration of Holliday junction DNA, which is the recombination intermediate. RuvA is the Holliday junction specific DNA binding protein and RuvB functions as the ATP-dependent motor protein. Biochemical, electron microscopic and X-ray crystallographic studies show crucial amino acid residues for RuvA and RuvB to promote branch migration, and the reaction mechanism of branch migration of Holliday junction in details. In this study, we have constructed the single-molecule system to observe the RuvARuvB directed translocation of Holliday junction, in order to clarify the reaction mechanism of branch migration of Holliday junction in more details. Based on the obtained results, we will discuss about the mechanism of the RuvA-RuvB mediated branch migration of Holliday junction. 